\title{
OPEN Value of clinical, ultrasonographic and MRI signs as diagnostic differentiators of non-benign lipomatous tumours
}

Karishma Khan ${ }^{1,2}$, Elayne Azzopardi ${ }^{1 \bowtie}$, Liberato Camilleri® 4 , Ernest A. Azzopardi1,3,4,5,5 \& Thomas H. Bragg ${ }^{1,5,5}$

Suspicion of malignant change within a lipoma is a common and increasing workload within the UK Sarcoma multidisciplinary team (MDT) network, and a source of considerable patient anxiety. Currently, there is no lipoma-specific data, with regard to which clinical or radiographic features predict non-benign histology, or calculate an odds-ratio specific to a lipomatous lesion being nonbenign. We performed a 9-year, double-blind, unmatched cohort study, comparing post-operative histology outcomes (benign versus non-benign) versus 15 signs across three domains: Clinical (size of tumour, depth, growth noticed by patient, previous lipoma, patient felt pain), Ultrasonographic (size, depth, vascularity, heterogenous features, septae) and MRI (size, depth, vascularity, heterogenous features, septae, complete fat signal suppression). Receiver operating characteristic (ROC) analysis, odds ratios and binary logistic regression analysis was performed double-blind. When each sign is considered independently, (ROC analysis, followed by binary logistic regression) only Ultrasound depth is a significant predictor $(p=0.044)$ of a histologically non-benign lipoma. Ultrasonographically determined vascularity and septation were not statistically significant predictors. None of the clinical signs were statistically significant $(p>0.05)$. Of the MRI signs none was statistically significant $(p>0.05)$. However, heterogeneous MRI features fared better than MRI depth. Ultrasound signs (Pseudo R-Square $=0.105$ ) are more predictive of the post-operation histology outcome than Clinical signs (Pseudo R-Square $=0.082$ ) or MRI tests (Pseudo R-Square $=0.052$ ) Ultrasound and Clinical tests combined (Pseudo R-Square $=0.147$ ) are more predictive of the post-operation histology outcome than MRI tests (Pseudo R-Square $=0.052$ ). This work challenges the traditional perception of "redflag" signs when applied to lipomatous tumours. We provide accurate data upon which an informed choice can be made, and provides a robust bases for expedited risk/benefit. The importance of an experienced and cohesive MDT network is emphasised.

Tumors of adipose represent both the commonest (50\%) of all benign mesenchymal tumours as lipomas, and the commonest group of malignant mesenchymal neoplasms (liposarcomas), which incurs a considerable disease burden and healthcare $\operatorname{cost}^{1,2}$. It is therefore surprising that there is no literature that explores the value of clinical, ultrasonoraphic, and magnetic resonance imaging signs in current clinical use, alone or in combination, specifically in differentiating benign from non-benign adipose tumours.

The UK National Institute for Health and Care Excellence (NICE) recognises the difficulty in distinguishing benign from malignant tumours as the principal problem in their management ${ }^{3}$. Discerning benign from malginant is the key decision that impacts on morbidity, mortality, and service delivery ${ }^{4}$. Besides ultrasonograpy and magnetic resonance imaging, current consensus opinion both by NICE and the British Sarcoma Group advocates any lump with any size, or size $>5 \mathrm{~cm}$, or deep to the deep fascia, or painful is considered higher risk for potential malignancy until proven otherwise, as a blanket approach to any soft tissue sarcoma ${ }^{5,6}$.

${ }^{1}$ The Welsh Centre for Burns and Plastic Surgery, Heol Maes Eglwys, Swansea SA66NL, UK. ${ }^{2}$ Cardiff University Medical School, Heath Park, Cardiff CF14 4XY, UK. ${ }^{3}$ University College London, London, UK. ${ }^{4}$ University of Malta Tal-Qroqq Campus, Malta GC. EU, UK. ${ }^{5}$ These authors jointly supervised this work: Ernest A. Azzopardi and Thomas H. Bragg. ${ }^{\boxplus}$ email: Laser.surgeon@yahoo.com 
Study aims. To determine the diagnostic value of 15 signs across three domains, as used in standard sarcoma multidisciplinary team (MDT) practice, for primary tumours of adipose: Clinical (size of tumour, depth, growth noticed by patient, previous lipoma, new onset of pain), Ultrasonographic (size, depth, vascularity, heterogenous features septae) and MRI (size, depth, vascularity, heterogenous features, septae, complete fat signal suppression).

Hypothesis That current clinical, ultrasonographic and magnetic resonance signs do not equally predict and differentiate benign from non-benign lipomatous tumours.

Operational definitions. We adopted A pragmatic, benign versus non-benign, binary approach, in keeping with World Health Organisatiion (WHO) defnitions ${ }^{7}$.

Gold standard was determined as the post-operative laboratory report histopathology, subject to standard UK National Health Service quality control criteria, and reported by an experienced validated MDT histopathologist.

"Benign" was defined as a lipoma, confirmed as benign on histologic report. "Non-benign" was determined as liposarcoma, including atypical lipomatous tumour/well-differentiated liposarcoma (which includes the adipocytic, sclerosing, inflammatory and spindle cell variants); de-differentiated liposarcoma; myxoid liposarcoma; and pleomorphic liposarcoma ${ }^{7}$.

Red flag Signs: The classical red flag signs were taken as defined by the National Institute for Health and Care Excellence $(3,5)$. These are reproduced in scheme 1.

Scheme 1. Features suggestive of malignancy in a lump.

- Lump $>5 \mathrm{~cm}$

- Lump increasing in size

- Lump deep to the fascia

- Pain.

Inclusion criteria. Patients with both histology, ultrasonographic or magnetic imaging reporting, presenting with suspected non-syndromic tumours of adipose, operated within the Wales Sarcoma Service between 2010 and March 2018.

Blinding. Data was anonymised at source. Data coding and primary analysis was performed blind. Statistical analysis was performed blind by an independent bio-statistician.

Ethics. This study was performed using retrospective anlysis of audit data anonymised at source. This was confirmed using the UK Health Research Authority's online decisional analysis tool, and seconded by institutional board correspondence ${ }^{8,9}$. All analysis methods were carried out in accordance with governing institutional guidelines and regulations at Swansea University and affiliated hospitals ${ }^{10}$ (clinical audit/service evaluation data).

\section{Methods}

We performed a 9-year, single sarcoma network, double-blind, unmatched cohort study, comparing post-operative histology outcomes (benign versus non-benign) versus 15 signs across three domains: Clinical (size of tumour, depth, growth noticed by patient, previous lipoma, patient felt pain), Ultrasonographic (size, depth, vascularity, heterogenous features septae) and MRI (size, depth, vascularity, heterogenous features, septae, complete fat signal suppression). The data was collected from the Welsh Sarcoma Service and incorporates the work of a senior, validated MDT team working according to British Sarcoma Standards. Data coding was performed as per Supplementary material ${ }^{1}$.

For each parameter, sensitivity, specificity, positive predictive value, negative predictive value, was followed by Reported-Observer Curve (ROC) analysis. Binary Logistic Regression Analysis was then used to investigate the collective contribution of signs in each domain area. Odds ratios were then calculated. Statistical significance was considered at $\mathrm{p}<0.05$. Data analysis was performed with SpSS for Windows (IBM Corp. Released 2017. Version 25.0. Armonk, NY: IBM Corp).

\section{Results}

Of 178 patients originally fulfilling inclusion criteria, histopathology reports were available in 106. Of these 25 lacked either ultrasonographic or magnetic resonance imaging data and were excluded, and the remainder (81) were included in the study (Fig. 1). To our knowledge this is the largest reported cohort of patients in the Literature to date.

For each domain, sensitivity, specificity, positive predictive value, and negative predictive value are reported in Table 1.

Clinical domain. Size of lipoma. ROC analysis shows this has some predictive power. However, p-value (0.516) exceeds the 0.05 level of significance. This is mainly attributed to the fact that the number of false positives (55) is quite large. 


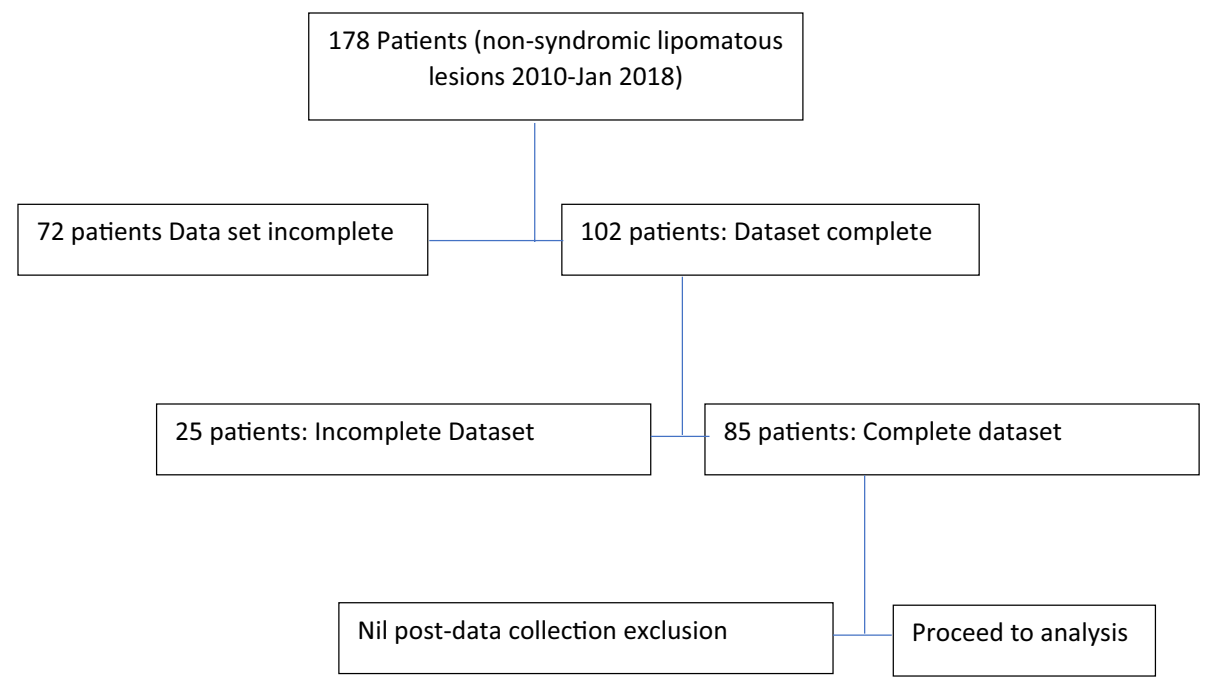

Figure 1. Attrition diagram to suppport exlusion of patients.

\begin{tabular}{|l|l|l|l|l|}
\hline Parameter & Sens (\%) & Spec (\%) & Ppv (\%) & Npv (\%) \\
\hline Clinical & 92.3 & 19.1 & 17.9 & 92.8 \\
\hline Size $>5 \mathrm{~cm}$ & 53.85 & 66.18 & 23.33 & 88.24 \\
\hline Depth & 38.46 & 39.71 & 10.87 & 77.14 \\
\hline Growth & 23.08 & 73.53 & 14.29 & 83.33 \\
\hline Pain & 15.38 & 91.18 & 25.00 & 84.93 \\
\hline Previous lipoma & \multicolumn{5}{|l|}{} \\
\hline Ultrasonographic & 61.54 & 31.34 & 14.81 & 80.77 \\
\hline Size >5 cm & 46.15 & 75.76 & 27.27 & 87.72 \\
\hline Depth & 33.33 & 77.61 & 21.05 & 86.67 \\
\hline Vascularity & 7.69 & 92.54 & 16.67 & 83.73 \\
\hline Heterog. features & 15.38 & 94.03 & 33.33 & 85.14 \\
\hline Septation & 66.67 & 9.76 & 13.95 & 57.14 \\
\hline MRI & 55.56 & 56.10 & 21.74 & 85.19 \\
\hline Size >5 cm & 0 & 85.36 & 0 & 79.55 \\
\hline Depth & 33.33 & 82.93 & 30.00 & v85.00 \\
\hline Vascularity & \multicolumn{5}{|l}{} \\
\hline Heterog features & \multicolumn{5}{|l|}{} \\
\hline
\end{tabular}

Table 1. Sensitivty, specificity, positive and negative predictive value for each parameter individually. Sens: sensitivity; spec: specificty; PpV: Postive predictive value; NpV: negative predictive value; heterog: heterogenous.

Depth of lipoma. The area under the ROC curve (0.600) exceeds the area under the 45-degree line (0.5) indicating that the depth of tumour has some predictive power. However, the p-value $(0.255)$ exceeds the 0.05 level of significance indicating that this area is not significantly larger than 0.5 . This is mainly attributed to the fact that the number of false positives (23) is quite large.

Growth noted by patient. The area under the ROC curve (0.391) is less than the area under the 45-degree line $(0.5)$ indicating that growth noticed by patients has no predictive power. This is mainly attributed to the fact that the number of false negatives (8) and false positives (41) are larger than the true positives (5) and true negatives (27).

Previous lipoma. The area under the ROC curve (0.533) exceeds the area under the 45-degree line (0.5) indicating that previous lipoma has some predictive power. However, the p-value $(0.709)$ exceeds the 0.05 level of significance indicating that this area is not significantly larger than 0.5 . This is mainly attributed to the fact that the number of false negatives (11) is quite large. 


\begin{tabular}{|l|l|}
\hline Parameter & Odds ratio \\
\hline lump $>5 \mathrm{~cm}$ & $\begin{array}{l}\text { If size of tumour is } 5 \mathrm{~cm} \text { or more, the odds that post-operation histology yields a non-benign } \\
\text { tumour is } 3.633 \text { times when size of tumour is less than } 5 \mathrm{~cm}\end{array}$ \\
\hline Tethered to fascia or deeper structures & $\begin{array}{l}\text { If the tumour includes fascia or is deep to fascia, the odds that post-operation histology yields a } \\
\text { non-benign tumour is } 2.257 \text { times when tumour depth is superficial }\end{array}$ \\
\hline Previous lipoma & $\begin{array}{l}\text { If previous lipoma is in the same position, the odds that post-operation histology yields a non- } \\
\text { benign tumour is } 3.341 \text { times when no previous lipomas are reported }\end{array}$ \\
\hline
\end{tabular}

Table 2. Odds of a non-benign histology for each clinical sign evaluated.

\begin{tabular}{|l|l|}
\hline Parameter & Odds ratio \\
\hline Ultrasound tumour depth & $\begin{array}{l}\text { If ultrasound tumour depth is fascial or sub-fascial, the odds that post-operation histology yields a non-benign } \\
\text { tumour is 4.059 times when ultrasound depth is superficial }\end{array}$ \\
\hline Vascularity & $\begin{array}{l}\text { If the tumour displays vascularity, the odds that post-operation histology yields a non-benign tumour is } 2.471 \\
\text { times when tumour does not invade microvasculature }\end{array}$ \\
\hline Septation & $\begin{array}{l}\text { If ultrasound detected septae are present, the odds that post-operation histology yields a non-benign tumour is } \\
1.394 \text { times when septae are not present }\end{array}$ \\
\hline
\end{tabular}

Table 3. Odds of a non-benign histology for each clinical sign evaluated.

Pain. The area under the ROC curve (0.483) is less than the area under the 45 -degree line (0.5) indicating that the pain felt by patients has no predictive power. This is mainly attributed to the fact that the number of false negatives (10) is larger than the number of true positives (3).

Although none of three predictors are significant, depth of tumour is the better of the three, followed by size of tumour and previous Lipoma. When the above three parameters we investigated collectively as potential predictors using a binary logistic regression model, the parameters explain only $8.2 \%$ of the post-operation histology outcome. The Odds ratios are reported in Table 2.

Ultrasound domains. Ultrasonographic size: The area under the ROC curve (0.464) is less than the area under the 45 -degree line (0.5) indicating that size of ultrasound has no predictive power. This is mainly attributed to the fact that the number of false positives (46) is large.

Ultrasonographic depth: The area under the ROC curve (0.610) exceeds the area under the 45-degree line (0.5) indicating that tumours occuring adjacent to or deep to fascia has some predictive power. However, the $\mathrm{p}$-value (0.214) exceeds the 0.05 level of significance indicating that this area is not significantly larger than 0.5 . This is mainly attributed to the fact that the number of false positives (16) is quite large.

Ultrasonographic evidence of tumour vascularity: the area under the ROC curve (0.555) exceeds the area under the 45-degree line (0.5) indicating that the ultrasound vascularity has some predictive power. However, the p-value (0.548) exceeds the 0.05 level of significance indicating that this area is not significantly larger than 0.5. This is mainly attributed to the fact that the number of false positives (15) is quite large.

Ultrasonographic evidence of tumour heterogeneity: The area under the ROC curve (0.501) exceeds the area under the 45 -degree line (0.5) by a very small margin indicating that the ultrasound heterogeneous feature has very little predictive power. Moreover, the p-value (0.990) exceeds the 0.05 level of significance indicating that this area is not significantly larger than 0.5 . This is mainly attributed to the fact that the number of false negatives (12) is quite large.

Ultrasound detection of septae: The area under the ROC curve (0.547) exceeds the area under the 45-degree line (0.5) indicating that the ultrasound detected septae has some predictive power. However, the p-value $(0.593)$ exceeds the 0.05 level of significance indicating that this area is not significantly larger than 0.5 . This is mainly attributed to the fact that the number of false negatives (11) is quite large.

Binary logistic regression of ultrasonographic domains. Logistic regression analysis was used to investigate the collective contribution of Ultrasound depth, ultrasound detected vascularity and ultrasound septae in predicting the outcome (non-benign, benign) of the post-operation histology. This three-predictor logistic regression model explains $10.5 \%$ of the post-operation histology outcome (Nagelkerke Pseudo $\mathrm{R}$-Square $=0.105)$. Moreover, ultrasound tumour depth is a significant predictor $(\mathrm{p}=0.044)$. Consequently it appears that ultrasound depth is the better of the three, followed by ultrasonographic evidence of vascularity and tumour septation.

The odds ratios, displayed in Table 3, all indicate that tumours of size $5 \mathrm{~cm}$ or more, tumours which abuts fascia or are deep to fascia and previous lipoma in the same position increase the risk that the post-operation histology yields a non-benign tumour since these odds are all larger than 1 . However, these odds ratios are not significantly larger than 1 because the p-values exceed the 0.05 level of significance. 


\begin{tabular}{|l|l|}
\hline Parameter & Odds ratio \\
\hline MRI depth & $\begin{array}{l}\text { If MRI depth involves fascia or reaches deep to fascia, the odds that post-operation histology yields a } \\
\text { non-benign tumour is } 1.708 \text { times when MRI depth is superficial to fascia }\end{array}$ \\
\hline MRI features are heterogeneous & $\begin{array}{l}\text { If MRI features are heterogeneous, the odds that post-operation histology yields a non-benign tumour is } \\
2.557 \text { times when MRI features are homogeneous }\end{array}$ \\
\hline
\end{tabular}

Table 4. Odds of a non-benign histology for each clinical sign evaluated.

MRI results. Size: The area under the ROC curve (0.382) is less than the area under the 45-degree line (0.5) indicating that MRI size has no predictive power. This is mainly attributed to the fact that the number of false positives (37) is large.

Depth: The area under the ROC curve (0.558) exceeds the area under the 45-degree line (0.5) indicating that the MRI depth has some predictive power. However, the p-value (0.587) exceeds the 0.05 level of significance indicating that this area is not significantly larger than 0.5 . This is mainly attributed to the fact that the number of false positives (18) is quite large.

MRI detected vascularity: The area under the ROC curve $(0.427)$ is less than the area under the 45 -degree line (0.5) indicating that MRI detected tumour vascularity has no predictive power. This is mainly attributed to the fact that the number of false negatives (9) is large.

MRI heterogenous features: The area under the ROC curve (0.581) exceeds the area under the 45-degree line (0.5) indicating that the MRI evidence of heterogeneous features have some predictive power. However, the p-value (0.449) exceeds the 0.05 level of significance indicating that this area is not significantly larger than 0.5$)$. This is mainly attributed to the fact that the number of false negatives (6) is quite large.

MRI Septae: The area under the ROC curve (0.409) is less than the area under the 45-degree line (0.5) indicating that MRI evidence of septation has no predictive power. This is mainly attributed to the fact that the number of false negatives (8) is large.

MRI fat suppression: The area under the ROC curve (0.336) is less than the area under the 45-degree line (0.5) indicating that Fat completely suppressed has no predictive power. This is mainly attributed to the fact that the number of false negatives (8) and false positives (18) are large.

Logistic regression for MRI criteria. Logistic regression analysis was used to investigate the collective contribution of MRI depth and MRI heterogeneous features in predicting the outcome (non-benign, benign) of the post-operation histology. This two-predictor logistic regression model explains $5.2 \%$ of the post-operation histology outcome (Nagelkerke Pseudo R-Square $=0.052$ ). Although none of these two predictors are significant, MRI heterogeneous features is the better of the two, followed by MRI depth.

Table 4 displays the odds ratios. The odds ratios all indicate that tumours that involve fascia or invades to fascia on MRI and heterogeneous features on MRI increase the risk that the post-operation histology yields a non-benign tumour since these odds are all larger than 1 . However, these odds ratios are not significantly larger than 1 because the $p$-values exceed the 0.05 level of significance.

Additionally, we used logistic regression analysis was used to investigate the collective contribution of size of tumour, depth of tumour, previous Lipoma, ultrasound depth, ultrasound tumour vascularity and ultrasound septae in predicting the outcome (non-benign, benign) of the post-operation histology. Now, this six-predictor logistic regression model now explains $14.7 \%$ of the post-operation histology outcome (Nagelkerke Pseudo $\mathrm{R}$-Square $=0.147$ ).

\section{Result summary}

$\begin{aligned} & \text { Of the clinical tests, depth of tumour is the best predictor of the post-operation histology outcome (non-benign, benign), followed by size } \\ & \text { of tumour and previous lipoma }\end{aligned}$
$\begin{aligned} & \text { Of the ultrasound tests, ultrasound depth is the best predictor of the post-operation histology outcome (non-benign, benign), followed by } \\ & \text { ultrasound tumour vascularity and ultrasound septae. Depth is also statistically significant on ROC analysis }\end{aligned}$
$\begin{aligned} & \text { Of the MRI tests, heterogeneous features on MRI is the best predictor of the post-operation histology outcome (non-benign, benign), fol- } \\ & \text { lowed by MRI depth }\end{aligned}$
$\begin{aligned} & \text { Of the clinical and ultrasound tests combined, ultrasound depth is the best predictor of the post-operation histology outcome (non-benign, } \\ & \text { benign), followed by size of tumour, ultrasound vascularity and previous lipoma }\end{aligned}$
$\begin{aligned} & \text { Ultrasound tests (Pseudo R-Square }=0.105) \text { are more predictive of the post-operation histology outcome than Clinical tests (Pseudo } \\ & \text { R-Square }=0.082 \text { ) and MRI tests (Pseudo R-Square =0.052) }\end{aligned}$
$\begin{aligned} & \text { Ultrasound and Clinical tests combined (Pseudo R-Square=0.147) are more predictive of the post-operation histology outcome than MRI } \\ & \text { tests (Pseudo R-Square }=0.052 \text { ) }\end{aligned}$

\section{Discussion}

The decision when to operate can be difficult with regard to tumours of adipose. An important aim in sarcoma management is early diagnosis and prompt referral ${ }^{11}$. Our study shows that the classical "red-flag" signs $s^{5}$ that are associated with malignant change are of limited value in the differentiation of lipomatous tumours. Much of the bases for the current "red flag symptoms" is based on the work of Johnson Pysent and Grimer"12. These authors used a weighting scheme to measure the likelihood of a malignant lesion and then converted this weight to a probability using the logistic function, where larger weights corresponded to higher probabilities of malignant 
lesions. Our work differs in serveral ways. Firstly, our approach is model-based where the contribution of each parameter is investigated individually using ROC curve analyses and then collectively with other parameters using Binary Logistic models. These models, which are appropriate for analyzing binary responses, yield odds ratios which measure the likelihood of non-benign histology in the presence of a parameter when compared to its absence. Moreover, we only included lipomatous tumours and data retrieval was double cross-checked. Statistical analysis was performed blind by an experienced statistician.

Only ultrasonographically determined tumour depth withstood binary logistic regression analysis to emerge as a significant predictor of the post-operative histology. Further, our results show that combined ultrasonographic and clinical examination, may be more predictive of post-operative histology when compared to magnetic resonance imaging in isolation. These results may also have implications on service delivery, access and co-production decision sharing. Patient anxiety and reduced quality of life often accompany the interim period between referral and final diagnosis. The odds ratios presented herein for each sign, contribute to informing decisions taken jointly between patient and the clinical team, and clarifying informed consent. In the presence of conflicting clinical, or radiological evidenced, these findings may facilitate MDT co-production decision making based upon the predictive strength of the individual signs within each domain. Further, the predictive superiority of combined clinical plus ultrasonographic examination, in particularly depth on ultrasound, have implications for service delivery and access. The Welsh Sarcoma Service now offers combined sarcoma screening clinics which are both rapid access and cost-saving compared to MRI imaging. Whilst the latter retains an important role in diagnostics, its use may be more focused on surgical planning and possible morbidity from surgery.

Limitations and direction of future research: Our findings are dependent on a caveat of a highly experienced sarcoma MDT being inolved in clinical examination. We also noted that some of the main effects (ultrasound depth, size of tumour, ultrasound vascularity and previous lipoma) yielded p-values that exceeded the 0.05 level of significance by a small margin. Even though, to our knowledge, this was the study with the largest study sample size reported in the literature to date, we cannot exlcude the possibility that these predictors could be significant if the sample size had to be increased further. It is known that when conducting hypothesis testing, the $\mathrm{p}$-value depends heavily on the sample size and it is very unlikely to attain statistical significance in the presence of heterogeneous responses.

These findings and their implications have been incorporated in the Welsh Sarcoma Service Multidisicplinary Meeting policy and form the bases our current practice.

Received: 24 August 2018; Accepted: 7 March 2019

Published online: 27 November 2020

\section{References}

1. Myhre-Jensen, O. A consecutive 7-year series of 1331 benign soft tissue tumours: clinicopathologic data Comparison with sarcomas. Acta Orthopaedica Scand. 52(3), 287-293 (1981).

2. Dei Tos, A. P. Liposarcomas: diagnostic pitfalls and new insights. Histopathology 64(1), 38-52 (2014).

3. National Institute for Clinical Excellence. Improving Outcomes for People with Sarcoma. 2006.

4. Abajo, B. M., Polo, J. R. T., Barrena, I. R. \& Poveda, M. D. Lipomas: classification, dermatologic manifestations and treatment. Int. J. Cancer Res. Prevent. $9(2), 125$ (2016).

5. Excellence NIfC. Suspected Cancer: Recognition and Referral. 2015.

6. Grimer R, Judson I, Peake D, Seddon B. Guidelines for the management of soft tissue sarcomas. Sarcoma. 2010;2010.

7. Fletcher, C. D. The evolving classification of soft tissue tumours-an update based on the new 2013 WHO classification. Histopathology 64(1), 2-11 (2014).

8. UK Health Research Authority. Defining research 2018. Available from: https://www.hra-decisiontools.org.uk/research/docs/Defin ingResearchTable_Oct2017-1.pdf.

9. UK Health Research Authority. Do I need NHS REC approval? 2018. Available from: https://www.hra-decisiontools.org.uk/ethic s/

10. Hughes J. Exemption from Research Ethics Committee Approval. In: Azzopardi DEA, editor. 2018.

11. Dangoor, A. et al. UK guidelines for the management of soft tissue sarcomas. Clin. Sarcoma Res. 6(1), 20 (2016).

12. Johnson, C., Pynsent, P. B. \& Grimer, R. J. Clinical features of soft tissue sarcomas. Ann. R. Coll. Surg. Engl. 83(3), 203 (2001).

\section{Author contributions}

K.K. participated in data retrieval and data processing. E.A. performed blinded data validation, and cross-checked the analysis. L.C. performed independent data analysis blind, contributed to manuscript write-up and redaction. E.A.A.: Supervised data retreival, analysis, main contributor to manuscript write up. T.B.: Originated the idea for the research. Supervised medical student. Imparted clinical experience as Wales Sarcoma MDT lead.

\section{Competing interests}

The authors declare no competing interests.

\section{Additional information}

Supplementary information is available for this paper at https://doi.org/10.1038/s41598-020-77244-2.

Correspondence and requests for materials should be addressed to E.A.

Reprints and permissions information is available at www.nature.com/reprints.

Publisher's note Springer Nature remains neutral with regard to jurisdictional claims in published maps and institutional affiliations. 
(c) (i) Open Access This article is licensed under a Creative Commons Attribution 4.0 International cc) License, which permits use, sharing, adaptation, distribution and reproduction in any medium or format, as long as you give appropriate credit to the original author(s) and the source, provide a link to the Creative Commons licence, and indicate if changes were made. The images or other third party material in this article are included in the article's Creative Commons licence, unless indicated otherwise in a credit line to the material. If material is not included in the article's Creative Commons licence and your intended use is not permitted by statutory regulation or exceeds the permitted use, you will need to obtain permission directly from the copyright holder. To view a copy of this licence, visit http://creativecommons.org/licenses/by/4.0/.

(C) The Author(s) 2020 